\title{
Intracranial Neurenteric Cysts: Two Atypical Cases and Review of the Literature
}

\section{Sonia Teufack, MD, Peter Campbell, MD, Yaron Moshel, MD, PhD}

Department of Neurological Surgery, Thomas Jefferson University, Philadelphia, PA

\section{Abstract}

\section{Introduction}

Neurenteric cysts (NCs) are rare intracranial lesions of endodermal origin. They typically arise as ventral intradural extramedullary spine developmental malformations. We present two atypical cases of intracranial NCs.

\section{Clinical Presentation}

The first patient presented with a headache and was found to have an enlarging $6.6 \mathrm{~cm}$ left frontal lobe cystic mass. The second presented with diplopia secondary to left third nerve palsy and was found a have $1.7 \mathrm{~cm}$ left superior prepontine lesion.

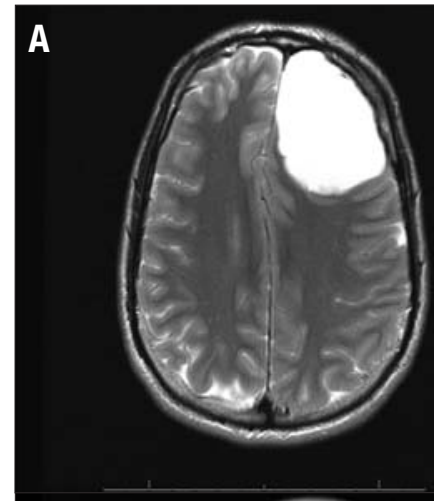

C

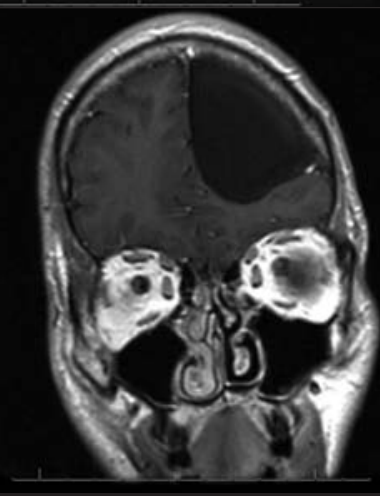

D

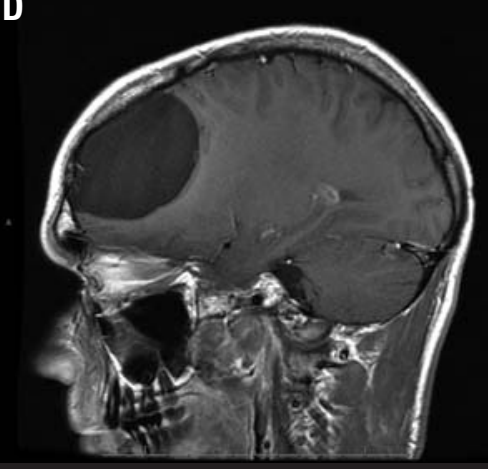

Figure 1

Brain MRI with a $4.7 \times 4.0 \times 3.2 \mathrm{~cm} 3$ extra-axial cystic structure adjacent to the anterior left frontal lobe; with mild effect on the adjacent brain. The cystic fluid has similar intensity to CSF on all sequences. (A)Axial T2 weighted; (B) Axial T1 post-contrast; (C) Coronal T1 post-contrast; (D) Sagittal T1 post contrast images.
Results

Both patients had the working diagnosis of arachnoid cyst. They respectively underwent a left frontal craniotomy and a transphenoidal endoscopic osteotomy for resection and fenestration of the cystic lesions. Histopathology evaluation revealed type A neurenteric cysts respectively with respiratory and gastrointestinal epithelia.

\section{Conclusions}

Although very rare, neurenteric cysts can occur intracranially.In recent years, the incidence of intracranial NCs has increased, possibly as a result of enhanced neuroimaging. It is thus crucial to become familiar with the presentation, radiographic and histopathologic characteristic and treatment options for NCs.

\section{Keyword \\ Neurenteric cyst}

\section{Introduction}

Neurenteric cysts are endothelium-lined structures thought to be derived from malformations involving embryonal rests of primitive endodermal cells. They were first described as intestinome by Puusepp in 1934 . Since then they have been called enterogenous cyst, enteric cyst, endodermal cyst, gastroenterogenous cyst, archentericcyst, and gastrocytoma. They typically arise as ventral intradural extramedullary spine developmental malformations. Neurenteric cysts (NCs) are rarely encountered intracarnially and represent about $0.01 \%$ of CNS tumors. Intracranial NCs generally present with symptoms of local mass effect or meningitis and in an infratentorial location. We present two atypical cases of NCs; one on the left frontal convexity and another in the interpeduncular cistern.

\section{Materials and Methods}

\section{Case 1}

A 27 year-old male presented to his primary care doctor with a chronic headache. An MRI of the brain revealed an extra-axial cystic structure adjacent to the anterior left frontal lobe, with mild mass-effect on the underlying brain. TheMRI signal appearance of the fluid within the NC follows CSF on all sequences, including 


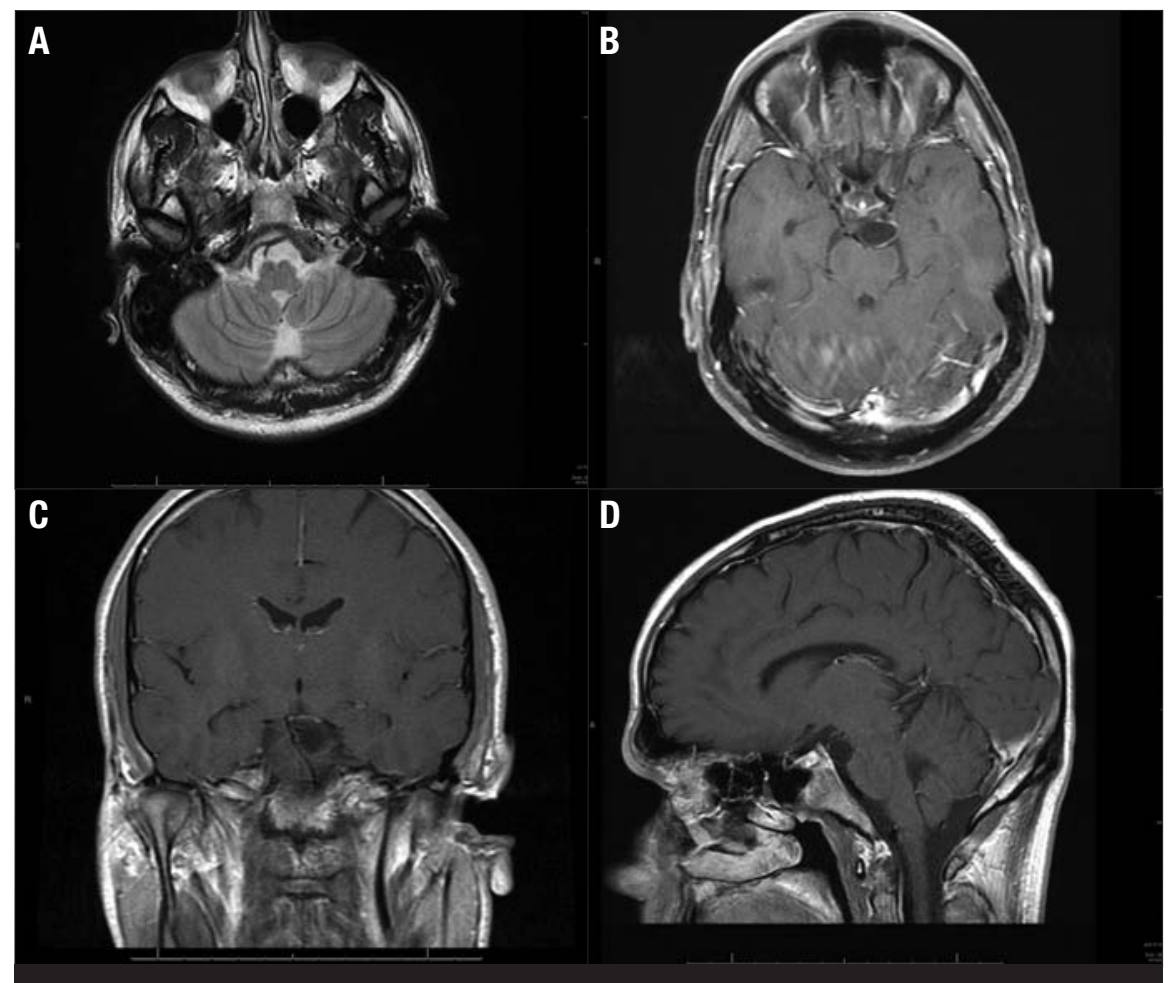

Figure 2

Brain MRI with a $1.7 \times 1.6 \times 1.3 \mathrm{~cm} 3$ cystic mass in the superior left prepontine cistern displacing the basilar artery to the right and impinging on the left fetal posterior communicating artery and third cranial nerve. (A) Axial T2 weighted; (B) Axial T1 post-contrast; (C) Coronal T1 post-contrast; (D) Sagittal T1 post contrast images.

FLAIR and diffusion weighted imaging. There were no areas of abnormal enhancement following intravenous contrast administration. Findings were consistent with an arachnoid cyst. Based on this incidental diagnosis, the clinical decision was to observe the lesion and follow the patient clinically.

Three years later, the lesion was noted to significantly enlarge on a follow-up MRI scan exerting a mild degree of mass effect on the surrounding brain in the left frontal lobe. The cyst fluid within the tumor continued to follow CSF signal characteristics on all sequences and had no enhancement. In addition, there was increased mass effect with subfalcine herniation and a $7 \mathrm{~mm}$ left to right midline shift. (Figure 1)

The patient underwent a left-sided craniotomy for excision and fenestration of the cystic lesion. The lesion was intradural, extra-axial, had a tan-white cystic membrane and clear fluid content. The brain parenchyma behind the lesion appeared normal. Histopathology of the cyst wall revealed respiratory epithelium, consistent with a neurenteric cyst. The patient did very well post-operatively; he remained neurologically intact and had resolution of his headache.

\section{Case 2}

A 30 year-old male presented with four months of headache and diplopia and on exam was noted to have a left pupil-involving third nerve palsy with ptosis; he had no other neurological deficits. A brain MRI revealed a moderately sized cystic mass in the superior left pre-pontine cistern displacing the basilar artery to the right and impinging on the left fetal posterior communicating artery and third cranial nerve. The lesion followed CSF signal on all pulse sequences and did not demonstrate restricted diffusion. (Figure 2)

The patient underwent an endoscopic endonasal transphenoidal transclival posterior cranial base approach including a pituitary transposition to get access to the retrosellardura overlying the inter-peduncular cistern.
Upon opening the dura, a grey capsule with a fine vascular network was identified between the left superior cerebellar artery (SCA) and posterior cerebral artery (PCA). The lesion was densely adhered to the SCA, basilar artery and their perforators. The cystic mass was removed in a piecemeal fashion. The posterior lateral aspect of the lesion was obscured by the paraclival carotid artery limiting access to the most lateral component of the lesion.

Post-operatively, the patient did well with no new neurological deficits. Pathology revealed a pseudo-stratified columnar epithelium with no goblet cells or cilia; the superficial layer was strongly positive for PAS and mucicarmine negative. Final diagnosis was Neurenteric cyst.

\section{Discussion}

Intracranial NE cysts are rare, benign lesions that have not been well recognized. Most cases of intracranial NCs involve the posterior fossa or craniovertebral junction. Supratentorial NCs are rare. In contrast, spinal NCs are usually found in the lower cervical and upper thoracic spinal cord and are extra-axial and lie ventral to the spinal cord. They can be associated with bony anomalies including cleft vertebrae, hemivertebrae and fused vertebrae. There are currently over 80 cases of intracranial NCs reported in the literature ${ }^{3}$, but only three cases of intra-axial supratentorial NCs.

The histopathology of NCs varies and can be divided in three categories; types A, B and C. Type A cysts resemble respiratory or gastrointestinal epithelium and are covered by a single or pseudostrtatified layer of ciliated or non-ciliated cuboidal or columnar epithelium on a basement membrane overlying fibroconnective tissue. Type B cysts are richer in connective tissue and can include smooth muscle, glandular and lymphoid tissues, and rarely nerve ganglia. Type $\mathrm{C}$ cysts resemble type $\mathrm{B}$, but they have glial elements in addition. NCs are differentiated from neuroepithelial cysts by the presence of a basement membrane, cilia, and goblet cells with secretory granules. Our two cases can be classified as Type A; case one resembled respiratory epithelium and case two resembled gastrointestinal epithelium.

The precise embryologic development of intracranial NCs is not clear. NCs are thought to result from the failure of the notochord and the gut to separate during formation of the alimentary canal. During the third and fourth weeks of embryogenesis, there is a transient communication between the primitive neural 


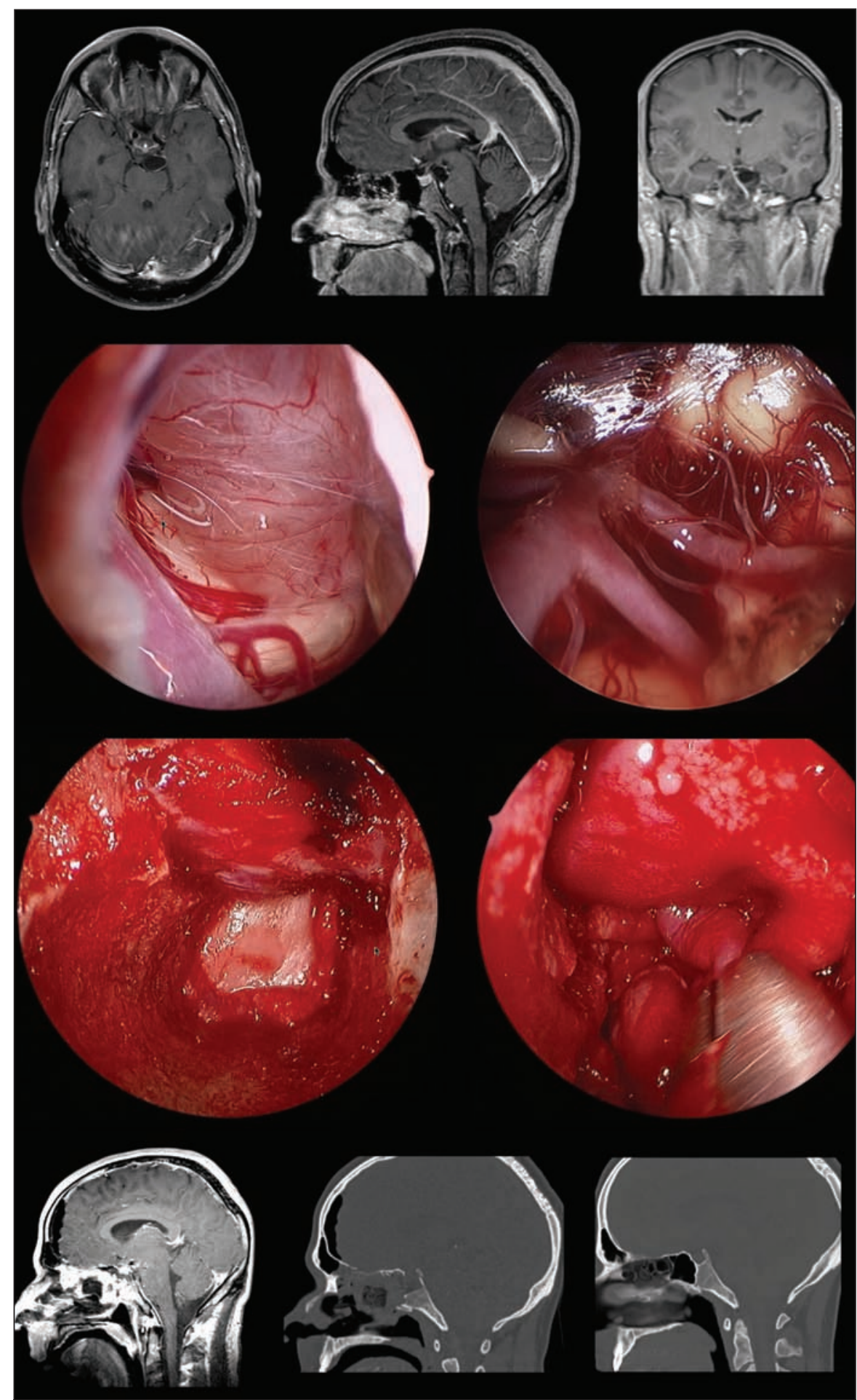

tube (ectoderm) and the enteric tube (endoderm) called the neurenteric canal. Later, the ectoderm derived notochord separates the embryonic ectoderm and the endoderm derived foregut. If the notochord develops abnormally, the endodermal cells become separated from the normal gut, and ectopic NE cysts begin to emerge intracranially. Although this theory explains the occurrence of spinal NCs caudal to the clivus, which is the cranial margin of the endoderm in embryos, it does not explain the presence of intracranial NCs.

Several authors have speculated about the embryological origins of intracranial NCs. Cheng et al. suggest that they result from rostral closure of the notochord by the mesenchyme forming the clivus. Alternatively, Graziani et al. suggest that like colloid and Rathke's cleft cysts, intracranial NCs are remnant of Seessel's pouch, a transient out-pouching of the oropharyngeal membrane at the cranial end of the foregut. The later theory is supported by the fact that NCs share several immuno-histo-chemical staining characteristics of Rathke's and colloid cyst. Neurenteric cyst are positive cytokeratin, EMA, CEA, CA 19-9, negative GFAP; Rathke's and colloid cyst may be positive cytokeratin, EMA, CEA, but are negative GFAP and CA 19-9. ${ }^{2,11}$ However, it does not explain laterally positioned intracranial NCs.

A review of previously published literature showed that the average age of diagnosis of intracranial NCs was 34 years-old ; which correspond to our patients' age group. Patients typically present with signs of local mass effect such as cranial nerve palsies, myelopathy, and signs of meningitis from micro-leakage of the cystic content $t^{11}$. Our first patient only presented with headache and had not focal signs, despite the local mass effect and midline shift evident of neuroimaging. On the other hand, our second patient presented with a pupil involving third nerve palsy, consistent with local compression of the ipsilateral nerve.

The typical appearance of NCs on neuroimaging consist of a midline extra-axial posterior fossa cystic mass. They are frequently hypodense on CT scans; however they are occasionally hyperdense. Similarly, they may be hypo to hyper-intense on T1 and T2 MRI sequences, or appear to have mixed or compartmentalized density ${ }^{2,11}$. These discrepancies are thought to result from variation in protein and lipid content of the cyst and as a result of the type of endodermal epithelium lining the cavity. The differential diagnosis for NCs includes arachnoid cyst, epidermoid cyst, dermoid cyst, 
and other endodermal cysts such as Rathke's cleft cyst and colloid cyst. Our patients both had cystic content with CSF characteristics, and clear gross appearance. Jhawar et al. ${ }^{4}$ presented a case with a hyperdense, T1-hyperintense, and T2-hypointense intra-axial temporal NC. Their cyst content was machine oil like and their cyst wall was lined with pseudostratified columnar ciliated epithelium resting on a fibrocollagenous basement membrane seeded with foreign body granuloma. NCs do not typically demonstrate diffusion restriction on MRI, which differentiates them from epidermoid cyst; they might have trace enhancement of the cystic wall ${ }^{3,11}$.

Surgical excision has been the only effective treatment option thus far for symptomatic intracranial NCs. Complete resection of the cyst wall whenever possible is the optimal treatment modality. Occasional recurrences several years later have been reported with excisional biopsy, partial wall resection and cysto-subarachnoid shunting ${ }^{8}$. Patients must be followed with subsequent MRI scans, as recurrence is possible even with apparent gross total resection.

\section{Conclusion}

Neurenteric cysts are rare intracranial lesions or endodermal origin. We presented two atypical cases; a frontal convexity and pre- pontine NCs. As it is frequently the case with intracranial NCs, the patients were initially misdiagnosed with arachnoid cysts. Significant advancement have been accomplished in neuroimaging; rendering it more accessible and utilized as a diagnostic tool. Consequently, an increased number of previously underrecognized intracranial pathologies, such as NCs, are being discovered. We believe that with the increased incidence of a frontal convexity and a prepontine NC, it is crucial to become familiar with the presentation, radiographic and histopathologic characteristic and treatment options for NCs.

\section{References}

1. Puusepp M. Variete rare de teratome sousdural de la region cervicale (intestinome): Quadripleggie-Expation: Guerison complete. Rev Neurol. 1934;2:879-886.

2. Preece MT, Osborn AG, Chin SS, Smirniotopoulos JG. Intracranial neurenteric cysts: imaging and pathology spectrum. AJNR Am J Neuroradiol. 2006;27(6):1211-1216.

3. Tucker A, Miyake H, Tsuji M, et al. Neurenteric cyst of the lower clivus. Neurosurgery. 2010;66(1):E224-5. 10.1227/01. NEU.0000361998.93841.C3.

4. Jhawar SS, Nadkarni T, Goel A. Intraparenchymal temporal neurenteric cyst. J Clin Neurosci. 2011;18(3):415-417. 10.1016/j.jocn.2010.04.046.

5. Kachur E, Ang LC, Megyesi JF. sIntraparenchymal supratentorial neurenteric cyst. Can J Neurol Sci. 2004;31(3):412-416.

6. Asamoto $\mathrm{S}$, Sugiyama H, Doi H, et al. A case of neuroaxis endodermal cyst]. No To Shinkei. 1999;51(6):520-523.
7. Bejjani GK, Wright DC, Schessel D, Sekhar LN. Endodermal cysts of the posterior fossa. Report of three cases and review of the literature. J Neurosurg. 1998;89(2):326-335. 10.3171/ ins.1998.89.2.0326.

8. 8. Shakudo M, Inoue Y, Ohata K, Tanaka S. Neurenteric cyst with alteration of signal intensity on follow-up MR images. AJNR Am J Neuroradiol. 2001;22(3):496-498.

9. Gumerlock MK. Epidermoid, dermoid, and neurenteric cysts In: Winn RH, ed. Youmans Neurological Surgery. Vol 1. 5th ed. Philadelphia, PA: WB Saunders; 2004:1223-1230.

10. Cheng JS, Cusick JF, Ho KC, Ulmer JL. Lateral supratentorial endodermal cyst: case report and review of literature. Neurosurgery. 2002;51(2):493-9; discussion 499.

11. 11. Graziani N, Dufour H, Figarella-Branger D, Donnet A, Bouillot P, Grisoli F. Do the suprasellar neurenteric cyst, the Rathke cleft cyst and the colloid cyst constitute a same entity? Acta Neurochir (Wien). 1995;133(3-4):174-180.

\section{Corresponding Author}

Yaron Moshel, MD, PhD

Department of Neurological Surgery

Thomas Jefferson University

909 Walnut St, 2nd Floor

Philadelphia, PA 19107

Email: yaron.moshel@jefferson.edu

Phone: 215-503-7000

Fax: 215-503-9170

\title{
Brain power
}

\author{
感 Jefferson. \\ Hospital for Neuroscience
}

1-800-JEFF-NOW

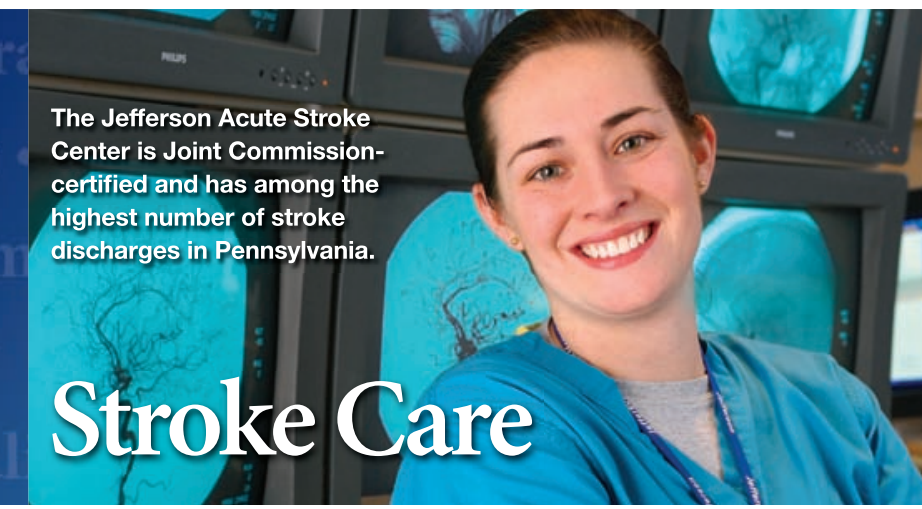

